\title{
La industrialización y el desarrollo regional en el Tercer Mundo, con especial referencia al caso de Brasil*
}

\author{
Michael Storper**
}

El argumento central de este artículo es que los diversos enfoques teóricos con los que se ha abordado la problemática del desarrollo del Tercer Mundo han exagerado la influencia de las fuerzas imperialistas en los fracasos de las políticas de industrialización y de desarrollo de los países subdesarrollados. En la primera parte, se hace un análisis retrospectivo tomando como ejemplo la experiencia de algunos países en desarrollo, señalando que los analistas no evaluaron correctamente la influencia de la diversidad de respuestas locales y tampoco tomaron en cuenta el impacto de las relaciones de clase, la ideología política y las políticas nacionales. Ello con el objeto de destacar que en la actual coyuntura económica mundial, en la que se está forjando una nueva estructura del sistema capitalis$t a$, bajo el predominio de la industrialización mediante sistemas de "producción flexible", resulta imperativo no permitir que el discurso teórico sobre el imperialismo desvíe la atención que debe prestarse a la función capital de la dinámica local contemporónea.

\section{Lecciones del pasado; realidades del presente}

El imperialismo ha sido el tema central, durante los últimos decenios, de muchos de los análisis especializados sobre el desarrollo del Tercer Mundo en general y sobre el desarrollo económico regional en particular. Ha asumido diversos aspectos, desde el del análisis de los sistemas mundiales hasta el de la teoría de la dependencia, pasando por el de la preocupación por las corporaciones

* Ponencia preparada para su presentación en la Conferencia Internacional Trends and Challenges of Urban Restructuring, Rio de Janeiro, 26-30 de septiembre de 1988. Traducción del inglés de Mario A. Zamudio.

** Escuela de Planificación Urbana, Universidad de California, Los Angeles. Agradezco la ayuda de la Fundación German Marshall, en la que era investigador mientras escribía esta ponencia.

Los datos sobre Brasil utilizados para ilustrar mi argumentación los tomé de un proyecto de investigación iniciado cuando trabajaba en Brasil como investigador de la Fundación Fulbright en 1986. También quisiera dar las gracias a las Comisiones Fulbright de Estados Unidos y Brasil y al Departamento de Economía de la Universidad de São Paulo por su ayuda, así como al Departamento de Geografía de la Universidad Federal de Santa Catarina (Florianopolis) por guiarme en mis visitas de campo a las industrias del sur de Brasil. 
trasnacionales y por el de las muchas variedades de teorías centradas en la función del comercio en el desarrollo nacional y regional. En la primera parte de este artículo, trataré de demostrar que la participación de las fuerzas imperialistas en los muchos fracasos de las políticas de industrialización y de desarrollo del Tercer Mundo durante los años sesenta y setenta ha sido frecuentemente exagerada. En ese proceso, los especialistas han pasado por alto a menudo el hacer un análisis cuidadoso de la diversidad de respuestas locales a las fuerzas mundiales, de las grandes diferencias en los resultados obtenidos del desarrollo y, asimismo, de la función que han tenido, en los países en desarrollo y en las regiones mismas, las relaciones de clase, la ideología política y las políticas nacionales. Con todo, mi propósito no consiste simplemente en hacer una crítica basada en el beneficio que proporciona la observación retrospectiva, sino en valerme de un breve examen del pasado como punto de partida para mostrar, en la segunda parte del artículo, que, en la actual coyuntura del desarrollo, no debemos desperdiciar esa lección. A partir del inicio de los años setenta, se ha estado forjando un nuevo periodo del desarrollo del sistema capitalista, periodo que se caracteriza por una competencia intenșificada en el comercio y en los mercados mundiales y por la desintegración del dominio económico estadunidense. Dicho periodo trae consigo un nuevo paradigma dominante de industrialización cuyo síntoma principal es el predaminio de los sistemas de "producción flexible", en lugar de los antiguos sistemas de producción en serie. Lo paradójico es que, de cierta manera, esa nueva estructura mundial pone de relieve la importancia de las respuestas locales (nacionales y regionales) a las oportunidades y peligros que trae consigo el desarrollo.

La geografía histórica de las sociedades capitalistas va siempre un paso adelante de la capacidad de nuestra colectividad para aprehender esa realidad. No obstante, la tarea de entender y -esperamos- moldear los procesos de desarrollo económico urbano y regional debería, en condiciones ideales, verse acelerada por el perfeccionamiento de teorías que nos permitieran hacer frente a nuevas y más detalladas circunstancias empíricas, dándonos una idea de dónde buscar y qué buscar. Para poder hacer frente a las realidades de la competencia y la industrialización posfordistas, resulta imperativo no permitir que el discurso teórico sobre el imperialismo desvíe la atención que debe prestarse a la función capital de la dinámica local contemporánea.

Antes de continuar con el argumento central, se impone una palabra acerca del método. Como enfoque principal sobre el desarrollo urbano y regional, adoptaré el proceso del cambio económi- 
co y, particularmente, la función de la industrialización. La producción es una fuerza motriz esencial en todas las economías capitalistas, y la dinámica de organización, tecnología y ubicación de la producción sigue siendo clave para entender el desarrollo a todas las escalas geográficas. ${ }^{1}$

\section{Para alejarnos de la teoría del imperialismo}

\section{Una no tan sentimental remembranza del pasado}

La historia de las políticas de industrialización y desarrollo del Tercer Mundo a partir de los años cincuenta y hasta los setenta es tan bien conocida que sólo requiere un breve recordatorio. En los grandes países como Brasil y México, la modernización industrial fue emprendida generalmente mediante la política de la sustitución de importaciones. Se esperaba que la diversificación forzada de los aparatos industriales de esos países aceleraría la llegada del desarrollo autosostenido. Tal expectativa fue extraída de las teorías de Perroux - quien argumentaba que las tecnologías definen "espacios económicos" consistentes en actividades estrechamente vinculadas entre sí- y Schumpeter -quien sostenía que el progreso económico consiste en la creación y solución de diversas clases de desequilibrios tecnológicas y de organización-. Así, la sustitución de importaciones introduciría primero tales desequilibrios para después solucionarlos mediante el establecimiento de complejos industriales que abarcarían el total de cada espacio económico. Finalmente, ciertos países, como Brasil, pusieron en práctica generalizada versiones regionales de esas políticas, administradas por una gran variedad de poderosas instituciones de desarrollo regional.

En general, las distintas corrientes ideológicas estaban de acuerdo respecto al mérito de esas políticas, pero por razones di-

\footnotetext{
${ }^{1}$ En general, me basaré en dos categorías marxistas clásicas para organizar mi argumentación: a saber: las fuerzas de producción y las relaciones de producción. Las primeras se refieren a los procesos que gobiernan la instalación de diversos tipos de industrias (en cuanto sistemas de empleo y de producción realizada físicamente) en ciertos tipos de espacios en particular. Las segundas, por otra parte. se refieren a las relaciones sociales que controlan las acciones de los capitalistas y de los estados (en especial, ]a competencia intercapitalista y los procesos de inversión asociados a ella) y a las relaciones entre las clases, así como a la distribución del ingreso y a la dinámica del crecimiento que se desprenden de esas relaciones. La interacción de las fuerzas y de las relaciones de producción en el espacio y en el tiempo moldea profundamente la industrialización.
} 
ferentes. Comúnmente, la derecha daba su apoyo a los programas de modernización, siempre y cuando se permitiera el ingreso de las corporaciones trasnacionales para hacer el trabajo; la izquierda, por su parte, también daba su apoyo porque la sustitución de importaciones tenía una gran afinidad con la teoría de Trotsky sobre el "desarrollo combinado y desigual", la cual, a su vez, estaba fuertemente impregnada por la desviación produccionista de gran parte de las teorías marxistas tradicionales sobre el desarrollo. Además, ambos grupos convergían en su apoyo a las demandas locales de afirmación de los sentimientos nacionalistas tercermundistas de independencia económica.

El éxito de esas políticas fue impresionante en muchos sentidos. En países como Brasil, en un periodo de unos cuantos decenios y hasta el inicio de los años ochenta, el aumento de la producción industrial, de la diversificación de la economía industrial, de la complejidad y la gama de los productos manufacturados y de los niveles de ingreso nacional, fue espectacular. No obstante, desde el punto de vista de tal aumento cuantitativo del nivel de desarrollo, el periodo de los años ochenta fue prácticamente una "década perdida". Además, a todo lo largo del proceso, la trayectoria del desarrollo se caracterizó por enormes distorsiones sociales y económicas: empobrecimiento relativo y absoluto de grandes segmentos de la población; migración acelerada de las zonas rurales a las urbanas y creación de las "clases peligrosas" en las ciudades; ciclos macroeconómicos viciosos de auge repentino, hiperinflación y estancamiento con inflación; endeudamiento creciente; burocracias hipertrofiadas, y proyectos faraónicos, de infraestructura e industriales, con fondos prestados, mientras las necesidades básicas de la población seguían sin ser satisfechas. Como parte integral de ese proceso, surgieron enormes centros urbanos "hidrocefálicos" cuyo desarrollo sobrepasó ampliamente el de muchas otras regiones. Por éstas y otras razones, el modelo de industrialización entró en descomposición a principios de los años ochenta.

En todas partes, la derecha ha culpado de sus fracasos a las intervenciones políticas en sí mismas y, en su mayoría, ha favorecido los mercados libres - esto incluye tanto a las derechas nacionales como a las instituciones financieras internacionales-. La izquierda, por su parte, atribuye la culpa a las corporaciones trasnacionales, las cuales, afirma, son colectoras de utilidades que se rehúsan a reinvertir en los países donde producen y que explotan a los trabajadores del Tercer Mundo para reducir costos y aumentar sus utilidades en sus países de origen. No obstante, ambos lados convergen nuevamente en los años ochenta en torno a la respuesta nacionalista a la cuestión de la deuda, argumentando 
que los pagos de intereses inhiben la inversión local y reproducen la dependencia mediante la transferencia de los excedentes económicos al exterior. Ciertos grupos de las diversas burguesías nacionales (en especial en Brasil) están de acuerdo también con la izquierda sobre las ventajas de establecer protecciones mercantiles (e. g., la lei de informática brasileña) para industrias como la de alta tecnologia.

Una tercera respuesta, que no es exactamente ni de derecha ni de izquierda, se centra en las fuerzas de producción en sí mismas. En este caso me referiré a los teóricos de la tecnología adecuada, quienes argumentan, basándose en el análisis de Lewis (1954) en ese mismo sentido, que los fracasos de la modernización en gran escala se deben al excedente de mano de obra; sí bien, a diferencia de Lewis, aquéllos atribuyen el excedente casi por completo al uso de tecnologías importadas y concluyen que tales tecnologias deben rechazarse en favor de aquellas con requerimiento intensivo de mano de obra.

Reconozco que he caricaturizado posiciones que admiten muchos matices, pero no por ello es menos cierto que las tres se centran en e] efecto que las fuerzas externas ejercen sobre el subdesarrollo, ya sea mediante las desviaciones tecnológicas, la repatriación de utilidades, el sistema comercial internacional, los efectos de la inversión de capital internacional o las actividades de las organizaciones financieras internacionales.

\section{El resultado negativo: un contrapunto}

La explicación más plausible de por qué esos países nunca ingresaron en la fase de crecimiento y expansión macroeconómicos equilibrados es que nunca aumentaron los salarios a tal grado que los ingresos más altos hubiesen podido permitir la expansión del consumo de la población a un ritmo suficiente para apoyar una inversión industrial continua y estable (Lipietz, 1986; Storper, 1984). El resultado fue una trampa "keynesiana" clásica: se insertaron industrias altamente productivas en economías carentes de un crecimiento del ingreso que apoyara la expansión continua del consumo de los productos de esas industrias. En otras palabras, la industrialización basada en lạ sustitución de importaciones introdujo fuerzas de producción modernas en sociedades que carecían de relaciones sociales compatibles con dichas fuerzas. Algunos investigadores han llamado "fordismo periférico" a ese fenómeno (Lipietz, 1986).

¿Por qué ocurrió? Existen muchas interpretaciones opuestas. 
Entre ellas, algunas atribuyen la causalidad a la tecnología misma; en tal caso, afirman en su argumentación que una capacidad limitada de absorción de mano de obra deja enormes excedentes de esta última que entonces pueden ser explotados con facilidad y, en consecuencia, los salarios y el poder de compra permanecen perpetuamente restringidos. Sin embargo, es posible mencionar un gran número de argumentos lógicos correlativos de tal punto de vista.

- Primero: los que insistieron en adoptar esas tecnologías fueron, sobre todo, los responsables de las políticas de los propios países en vías de industrialización; ellos mismos buscaron con afán la sustitución de importaciones, a menudo en contra de los deseos de las trasnacionales.

- Segundo: algunos países han tenido éxito en la adaptación de tecnologías importadas a sus mercados y a las condiciones locales de suministro de factores, mientras que otros no. El primer caso puede ilustrarse mediante lo ocurrido con la industria automotriz japonesa durante el inicio del periodo de posguerra; en cambio, Brasil y México caen en la segunda categoría (Cosumano, 1985). En otras palabras, la adaptación tecnológica no es imposible, pero depende de agentes locales. Para que América Latina la hubiese logrado, habria sido necesario, por supuesto, que limitara la participación de las trasnacionales en el proceso de sustitución de importaciones, pero se trata de una decisión que sólo estuvo sustancialmente al alcance de los grandes países del Tercer Mundo en los años cincuenta.

- Tercero: la tecnología no es una deus ex machina; se requiere que los agentes sociales, los empleadores en este caso, aprovechen los mercados de mano de obra generados por las industrias que requieren una inversión intensiva de capital. Y, como se sabe, en lugares como Brasil, los verdaderos culpables a este respecto no son los empleadores de las grandes fábricas de propiedad directa de las corporaciones trasnácionales (que, en general, pagan los salarios más altos en los sectores industriales de esos países, si bien es cierto que siguen siendo muy bajos en comparación con los patrones salariales de los países desarrollados), sino las empresas de propiedad local, muchas de las cuales están vinculadas, velada o abiertamente, con las trasnacionales. Esas empresas tienen excedentes de mano de obra a los que común. mente explotan en una medida mucho mayor que sus socias trasnacionales y sólo en raras ocasiones ponen en práctica políticas significativas de protección de la mano de obra para impedir tal explotación (en diferentes épocas, Argentina y Chile han consti- 
tuido las únicas excepciones dignas de ser mencionadas).

- Cuarto: este punto se relaciona estrechamente con el anterior, ya que las burguesías nacionales y sus aliados de los sectores militares de países como Brasil, Argentina y Chile han sido responsables en una gran medida de la brutal represión de los movimientos laborales que podrían haber presionado con éxito para obtener salarios más altos. En Brasil, de hecho, las burguesías industriales nacionales y los intereses militares nunca han roto por completo con las élites terratenientes rurales, y estas últimas persiguen con afán intereses a menudo hostiles al desarrollo de una economía industrial urbana, madura y moderna. En Brasil, por ejemplo, existe un pacto tácito entre las élites industriales y las rurales para impedir la reforma agraria, la cual sería necesaria, según algunos, para provocar el flujo de mano de obra a las ciudades y así romper el círculo de bajos salarios urbanos y subdesarrollo industrial. Tanto las élites urbanoindustriales como las rurales obtienen beneficios mediante la reinversión de sus superutilidades en actividades devengadoras de rentas (como la especulación con bienes raíces] y, sin duda alguna, esas élites se verían amenazadas por una economía realmente más competitiva y menos oligopólica.

Vistas así las cosas, es difícil afirmar que los agentes sociales que han impedido activamente la realización del circuito ingresos-consumo, que habría sido necesario para un proceso de industrialización sostenido asociado a la sustitución de importaciones, son exclusivamente externos a esos países, y tampoco puede considerarse que sus acciones fueron dictadas por las necesidades abstractas del capitalismo internacional en los años cincuenta, sesenta y setenta.

Algunos analistas, en especial Evans [1979], han subrayado la acción conjunta de las trasnacionales, el Estado y el capital local en los procesos de desarrollo, y ponen un gran énfasis en la noción de que, en ciertas ocasiones, los intereses de los estados "desarrollistas" del Tercer Mundo y los de los capitalistas externos han convergido sustancialmente. Por ejemplo: la estrategia de la sustitución de importaciones fue una empresa completamente lógica durante los años cineuenta y sesenta, tanto para los intereses nacionales como para los extranjeros. El régimen de industrialización predominante en las economías avanzadas, esto es, la producción fordista en serie, poseía tales características tecnológicas que, en gran parte, pudo ser tomado e implantado en nuevos lugares con poca pérdida de eficacia, siempre y cuando se hubiese satisfecho cierta escala mínima de operaciones. Ello pudo hacerse 
así porque tanto los productos como los procesos habían entrado en una fase relativamente alta de uniformidad y estabilidad; el conocimiento de las economías dinámicas había sido uniformado e integrado a la maquinaria misma. También, las corporaciones trasnacionales y las economías nacionales más ricas (en especial Estados Unidos) se encontraban en una buena situación para transferir recursos al exterior: disfrutaban de altas utilidades y contaban con un sistema monetario internacional estable y con una competencia comercial extranjera relativamente reducida. Este último aspecto significa que una gran parte de sus inversiones en lugares como Brasil estaba destinada a ayudar a los mercados locales y no tan sólo -como a menudo se ha afirmado erróneamente- al uso de otros países para reducir costos de bienes que posteriormente eran reimportados por el Norte. ${ }^{2}$ Por lo tanto, las condiciones internacionales eran muy propicias para el éxito de las polfticas de sustitución de importaciones. Ahora bien, a pesar de esa lógica de los intereses convergentes, el comportamiento real fue frecuentemente el inverso: en muchos paises del Tercer Mundo, el capital nacional o el Estado nunca permitieron que florecieran las condiciones políticas y sociales internas necesarias para desencadenar un círculo positivo de salarios y consumo crecientes. Por lo demás, existen todas las razones para creer que las corporaciones trasnacionales razonables habrian preferido un mayor crecimiento del Tercer Mundo, ya que ello habría ampliado sus mercados, sin importar los altos salarios que se habrían visto obligadas a pagar a la mano de obra en sus fábricas instaladas en los países subdesarrollados.

Como puede verse con claridad, mi argumentación busca situar los orígenes de los fracasos del "fordismo periférico" de las últimas décadas, al menos en parte, en el tipo de políticas nacionales y relaciones de clase de los propios países en proceso de industrialización del Tercer Mundo. Esta actitud ha sido denominada recientemente como perspectiva "postimperialista", para denotar el nuevo énfasis que se hace en la función que tienen la complejidad, la diversidad y la importancia de las relaciones sociales locales (esto es, en las propias naciones en desarrollo) en el tipo de resul-

\footnotetext{
${ }^{2}$ La reubicación de la producción hacia el Tercer Mundo para reducir costos (mediante la reimportación de bienes terminados) no constituyó una estrategia particularmente importante para las empresas manufactureras trasnacionales hasta fines de los años setenta y sigue representando tan sólo una pequeña fracción de la producción industrial de los países desarrollados. Lo que ha crecido con mayor rapidez durante los últimos diez años es la competencia de importaciones de un país desarrollado a los otros.
} 
tados de las interacciones de esos países con las fuerzas trasnacionales (Becker y Sklar, 1987; Corbridge, 1988). A este respecto, me hago portador de un concepto expuesto antes por Cardoso [1977), quien ya desde entonces nos advertía que nos guardáramos de investigar los resultados históricos con una retrospectiva de demasiada inevitabilidad; y en ningún tipo de análisis se necesita con más énfasis que en el de los procesos de desarrollo aparentemente afectados en gran manera por las corporaciones trasnacionales o por un supuesto "capitalismo mundial". Aunque los países ricos y sus grandes corporaciones tienen ciertamente una parte de la responsabilidad por los fracasos y distorsiones de la industrialización que se han observado en los países que sustituyen importaciones, centrar la atención exclusivamente en las fuerzas externas nos impide hacer un análisis cuidadoso de los complejos y diversificados intereses estratégicos y capacidad política tanto de los grupos trasnacionales como de los nacionales. Esa actitud aviva a menudo la flama del nacionalismo económico, perpetuando así la peligrosa ilusión de la comunidad nacional orgánica en el Tercer Mundo, en lugar de la realidad de los intéreses materiales diferenciados que siempre están presentes en esas sociedades.

Estas observaciones parecen resurgir con cada nueva generación; pero a menudo son sepultadas bajo un torrente de invectivas respecto a los variados enemigos externos del desarrollo, ya sean otras naciones o estructuras abstractas. Como veremos, sería un grave error sepultarlas nuevamente en este momento en que el siglo llega a su fin.

La industrialización y la urbanización en el Tercer Mundo en los años 1960 y 1970: el problema de la primacía metropolitana

¿Qué significa todo ello para el análisis de la urbanización del Tercer Mundo durante el periodo considerado? Me centraré en una dimensión particular del fenómeno, la del llamado problema de la "hiperurbanización" y su contrapartida: las políticas para alentar la descentralización. Ese problema y esas políticas son aspectos importantes del paisaje geográfico y político en países como Brasil, México, Corea, Indonesia y Argentina.

El crecimiento de las grandes ciudades como São Paulo, México y Seúl, en las que se concentran altas proporciones de la población y la producción económica nacionales, se ha producido rápidamente de manera paralela a la industrialización forzada de las décadas recientes. No obstante, como ocurre en el caso del proceso de industrialización en general, a menudo se compren. 
den mal las razones precisas de la hiperurbanización y existe una tendencia a atribuir su causalidad a los objetivos más convenientes, esto es, la teconología moderna, las corporaciones trasnacionales y el capitalismo internacional, todos los cuales contribuyen de hecho a la hiperurbanización, pero, desde el punto de vista de la causalidad, no se relacionan internamente con ella.

Tomemos, por ejemplo, el caso de São Paulo, que es un enorme centro económico-industrial en cuya área metropolitana se lleva a cabo más del $60 \%$ de la producción industrial de Brasil. La región hace alarde de un ingreso per cápita dos veces más alto, en números redondos, que en el resto del país; en ella habitan las clases obreroindustriales y medias más numerosas y prósperas de Brasil y se ha convertido en una capital cultural e intelectual de gran vitalidad. Como en el caso de las otras ciudades importantes del Tercer Mundo, São Paulo cuenta también con una población en crecimiento continuo y con problemas generalizados de contaminación, marginalidad social y violencia. Los distritos industriales vecinos conocidos como "ABCD" (Saṇto Andre, São Bernardo do Campo, São Caetano do Sul y Diadema) constituyen realmente gigantescos centros de empleo industrial dominados por las fábricas de corporaciones trasnacionales como Volkswagen y General Motors. Sin duda alguna, las oportunidades de empleo de São Paulo crean un atractivo poderoso para inmigrantes de otras regiones del país y, así, contribuyen al incremento continuo en la escala de su urbanización. Los empleos en las fábricas de las corporaciones trasnacionales, por ejemplo, representan salarios generalmente muy por encima de los pagados en el resto de la economía manufacturera de São Paulo, para no mencionar el sector de servicios. No obstante, la responsabilidad por el tamaño de São Paulo no puede atribuirse exclusivamente a las trasnacionales, ya que los empleos que proporcionan, como quiera que sean medidos, constituyen una pequeña proporción del sector manufacturero, el cual, a su vez, representa sólo alrededor de un tercio del empleo total de la región. En São Paulo, un solo sector -el de la industria metálica- cuenta con más de 100000 establecimientos. Y en estas pequeñas empresas es donde se genera la mayor parte del empleo, a pesar de que, por lo común, sus niveles salariales representan una mera fracción de lo pagado por las trasnacionales. En otro estudio (Storper, 1984) afirmé que, en muchos casos, los niveles salariales típicos de la industria paulista no son sufícientemente más altos que en el resto del país para compensar siquiera las diferencias en el costo de la vida, mucho menos para proporcionar a la mano de obra una participación constante en la producción (es decir, la productividad es por lo común 
mucho más alta en Sāo Paulo, pero los salarios no comparten esa productividad en proporción similar con la mano de obra, dando así a los empleadores un excedente económico mayor en São Paulo que en el resto del país; véase Azzoni, 1986].

¿Por qué, entonces, sigue emigrando la gente a São Paulo? La respuesta debe residir en una serie de factores "de impulso", y éstos se encuentran principalmente en el campo, es decir, tanto en el desarrollo como en el subdesarrollo de las otras regiones de Brasil: en el desarrollo, porque los grandes procesos de modernización agrícola, a menudo brutales, han desplazado literalmente a millones de personas de la tierra; en el subdesarrollo, porque regiones enormes permanecen con niveles muy bajos de productividad, mientras que otras siguen caracterizándose por los altos niveles de concentración de la propiedad de la tierra típicos de los latifundios. Esos factores de impulso tienen sus complejos orígenès en el seno de la propia sociedad brasileña. Podemos citar algunos de esos orígenes: a) los coroneis del noreste brasileño, que durante siglos han constituido un poderoso bloque político y social de la formación social brasileña y su existencia tiene poco qué ver con el capitaljsmo industrial moderno; b) el fracaso persistente de cualquier reforma agraria significativa en Brasil (que podría permitir que una gran cantidad de personas permaneciera en la tierra, como alguna vez ocurrió en Corea], debido fundamentalmente a los bloqueos políticos a nivel nacional creados por las alianzas entre las élites rurales, los militares y una burguesía urbaoindustrial insuficientemente movilizada, indiferente $\mathrm{y}$, a veces, cómplice; c) el amplio apoyo a las ideologías geopolíticas nacionalistas de ocupación regional del Amazonas y la veneración ideológica respecto a las modernas tecnologías agrícolas promovida a muchos niveles del Estado y la sociedad. Aunque las corporaciones trasnacionales venden productos químicos y equipo a las granjas modernizadas, existen pocas pruebas de que ejerzan alguna influencia directa significativa en la formulación de las políticas de desarrollo rural de Brasil. Ciertamente, las instituciones financieras internacionales y los técnicos agrícolas de los países desarrollados cooperan en fomentar el proceso que impulsa a la gente fuera de la tierra, pero las pruebas sugieren que no son más que socios menores de las autoridades brasileñas del desarrollo.

Volviendo a los intentos por reducir el "impulso" de la pobla. ción hacia las grandes ciudades a través de la descentralización de la producción, es evidente que, en general, las trasnacionales han ido a la delantera de las compañías nacionales. Lo anterior puede explicarse con facilidad. Las trasnacionales, por ejemplo, cuentan por lo general con plantas de gran escala, sus procesos 
de producción son altamente uniformados, por lo que sus costos de transporte de insumos y productos por unidad son bajos, $\mathrm{y}$, finalmente, sus requerimientos de mano de obra son relativamente estables, todo lo cual, por ende, hace que su necesidad de estar cerca de un centro de oferta de mano de obra numerosa y flexible sea menor que en el caso de las empresas pequeñas. En efecto, la presencia de las trasnacionales es más fuerte en lugares muy alejados de São Paulo, como São Jose dos Campos, o en el anillo de ciudades como Americana, Rio Claro, Limeira, Piracicaba, etcétera, o-para poner un ejemplo más significativo- Fiat en Betim, en el eștado de Minas Gerais. Es evidente que, si hubiese suficientes apoyos infraestructurales disponibles, las trasnacionales serían felices de poder establecer sus plantas muy lejos de las áreas centrales tradicionales del país debido $\mathrm{a}$ su capacidad de vinculación a gran distancia y a que ello les permitiría pagar salarios incluso menores que en São Paulo y escapar a los sindicatos relativamente militantes establecidos ahí.

¿Por qué siguen existiendo presiones centralizadoras tan fuertes en la economía brasileña? En la nıayoría de los países que tienen sistemas urbanoindustriales más dispersos, las empresas y los trabajadores toman "decisiones" respecto a su lugar de establecimiento basadas en un conjunto de opciones y motivaciones diferentes de las que se encuentran presentes en Brasil (Storper, 1984]. En el caso de los países ricos, por ejemplo, si una alta proporción del proletariado industrial de una nación fuese concentrada en una sola ciudad, surgirían presiones salariales al alza, intolerables para los empleadores, debido a un gran número de razones: los efectos que ejercería sobre el costo de la vida el hecho de colmar el espacio con tantos consumidores; el surgimiento prácticamente seguro de una escasez de aprovisionamiento en el mercado de mano de obra local (debido a que es más difícil provocar la migración en un país donde no existen grandes núcleos de población rural desesperada), y la probable politización de una fuerza de trabajo concentrada espacialmente. Como ha ocurrido a menudo en el pasado, esas circunstancias llevarían a los empleadores a evitar los lugares centrales invirtiendo en otra parte, lo cual produciria, de todas maneras, un sistema urbanoindustrial más disperso geográficamente. En Brasil, no obstante, eso no ocurre porque tales diseconomías de aglomeración se ven impedidas por el excedente de mano de obra migrante y porque los intentos de organización del proletariado urbano son reprimidos, a menudo violentamente.

Existen otras razones que explican el hecho de que continúe la centralización, y se refieren al dinámica del desarrollo macro- 
económico en una economía industrializada y a su correlato, la sucesión sectorial (Storper y Walker, 1989): En los países más desarrollados, un ingreso y un consumo al alza proporcionan un ímpetu poderoso a la diversificación sectorial continua de la economía industrial, en la cual se desarrollan e introducen nuevos bienes que, con cierta frecuencia, acaban por imponer una producción en gran escala. Además, la gran cantidad de ingresos disponibles estimula el desarrollo de esos nuevos productos. ${ }^{3} \mathrm{~A}$ menudo, las nuevas industrias requieren, a su vez, insumos, mano de obra especializada y condiciones físicas e institucionales que no se encuentran presentes en las áreas industriales ya desarrolladas, por lo que es más probable que los nuevos sectores sean los que rompan con los patrones de ubicación existentes, ya que no son tan dependiéntes de los diversos tipos de mano de obra especializada o de los proveedores de insumos -en pocas palabras, de las economías (de aglomeración) externas-, desarrollados en los centros industriales existentes, como lo son las industrias ya establecidas en estos últimos. Cuando surgen nuevos sectores, se abre entonces una "ventana de oportunidades de ubicación" y, por ende, la posibilidad de que hagan su aparición nuevos centros geográficos de crecimiento (Scott y Storper, 1987). En los países desarrollados, la historia de los cambios espectaculares en los patrones de desarrollo regional puede narrarse en gran parte, en realidad, como la del comportamiento que ciertos grupos de industrias vinculadas entre si mostraban respecto al lugar a medida que surgían y crecían en importancia (Storper y Walker, 1989). Cada nuevo grupo de sectores dominantes traza su propio patrón geográfico creando distintos centros y periferias de crecimiento en lugares a menudo radicalmente diferentes de aquellos de las industrias que lo preceden. De hecho, el ascenso de São Paulo ha seguido esa trayectoria, ya que creció, hasta convertirse en el principal centro industrial de Brasil, cuando por primera vez se introdujeron en el país los sectores y métodos industriales modernos, liberándolo de la dependencia de las industrias y métodos artesanales tradicionales característicos de la en otra época dominante economía manufacturera regional de Rio (Cano, 1981).

Con todo, cuando la trampa antes descrita de los bajos ingresos para la clase trabajadora atrofia la expansión macroeconómica, es muy probable que la dinámica de la sucesión sec-

\footnotetext{
${ }^{3}$ También estimula el consumo de productos nuevos en las primeras fases de su desarrollo, cuando dichos productos son caros debido a que se fabricar, en pequeña escala mediante métodos relativamente artesanales y, también, con una mano de obra relativamente intensiva.
} 
torial se vea severamente retardada. Tal es incluso el caso de Brasil, que se encuentra entre las economías industriales más dinámicas del Tercer Mundo, y donde la sucesión sectorial y los aumentos en la escala de producción han sido insuficientes para, por una parte, provocar diseconomías de aglomeración importantes en São Paulo y, por la otra, para hacer posible la creación de nuevos centros de crecimiento. Consecuentemente, el origen del fracaso en lograr una mayor dispersión regional del desarrollo industrial puede rastrearse directamente hasta las limitaciones internas del propio modelo de desarrollo, como se describió en la sección anterior.

De esta afirmación se desprenden dos conclusiones. Primera, así como es necesario ir más allá de los análisis de los fracasos del desarrollo de los años sesenta y setenta que ignoran las relaciones de clase y las fuerzas políticas nacionales para dedicar una atención totalizadora a las fuerzas del imperialismo, también es necesario superar el punto de vista de los análisis de la urbanización y el desarrollo regional. Segundo, si se desea que la política de descentralización tenga éxito, ésta no puede ser formulada en los términos de una política espacial separada de la política económico-industrial en general. Brasil, por ejemplo, con poco éxito del cual jactarse y con un gran desperdicio de recursos, ha puesto en práctica durante más de tres decenios políticas generalizadas con miras a la descentralización geográfica del desarrollo -SUDENE, por ejemplo-. Las "catedrales en el desierto" - -esto es, plantas textiles y petroquímicas instaladas en los estados del noreste como Bahía y Rio Grande do Norte- tienen pocos vínculos comerciales con las regiones donde se encuentran, ya que continúan recibiendo la mayoría de sus insumos del sureste, y, lo que es peor, proporcionan un arma más para debilitar a la clase trabajadora del sureste, ya que los empleadores, apoyados por los subsidios gubernamentales, pueden emprender su producción en regiones periféricas que, de otra manera, no serían provechosas. Las plantas textiles instaladas en el noreste con tales subśidios, por ejemplo, pagan salarios más bajos que en el sur, y las empresas de esta última región hacen saber a su fuerza de trabajo que no existe la opción de la movilidad del capital. ${ }^{5}$ Por lo tanto, si se desea que la

\footnotetext{
" Traducción de "catedrale nell'deserto", término utilizado por los analistas italianos del desarrollo regional para describir los solitarios complejos petroquímicos instalados en el Mezzogiorno con pocos resultados provechosos.

${ }^{5}$ Fuente: entrevista con el gerente de planta de una gran empresa textil brasileña; Santa Catarina, noviembre de 1986. Las alusiones hechas en el texto fueron extraldas directamente de las estrategias de esa empresa, descritas en la entrevista.
} 
política de descentralización urbana y regional tenga éxito, no debe formularse independientemente de una política económico-industrial en la que se tomen en cuenta los factores geográficos.

Estas lecciones generales del pasado reciente son también muy pertinentes para las condiciones de la vida económica de los años ochenta, como lo veremos.

\section{Las estrategias de industrialización y desarrollo regional en los años ochenta}

El fin del posfordismo y el surgimiento de un nuevo sistema tecnológico-institucional

Los aparatos de producción capitalista pueden asumir muchas configuraciones tecnológicas e institucionales opcionales; a mediados del siglo XIX, por ejemplo, se vieron dominados por el clásico sistema de fábricas, mientras que, a mediados del $\mathrm{XX}$, predominó la producción en serie fordista. Cada configuración en particular consiste en un modelo de producción tecnológico-industrial determinado históricamente, que comprende una red de técnicas de producción, relaciones laborales, métodos de organización de la división de la mano de obra en cada una de las empresas y entre ellas mismas, relaciones administrativas y empresariales, etcétera. Cada modelo surge a la luz de las posibilidades tecnológicas disponibles en un momento en particular de la historia industrial junto con la estructura dominante de competencia capitalista (esto es, una gama de condiciones de mercado, estructuras de precios, el factor oferta/precios, etcétera). Como antes dijimos, a partir de los años cincuenta y hasta fines de los setenta, la estrategia de industrialización de muchos países estaba dirigida esencialmente hacia la transferencia de 'un modelo tecnológico-institucional, el de la producción en serie fordista, a los países más grandes y más ricos del Tercer Mundo; sin embargo, ese modelo ya no es funcional en grandes regiones del mundo y, lo que es más importante, las estructuras de competencia internacional que se desarrollaron en los años setenta y ochentá ya no permiten su reimplantación, todo lo cual ha modificado irrevocablemente las condiciones básicas de entorno para aplicar las estrategias de industrialización regional durante los años noventa, tanto en las economías en desarrollo como en las desarrolladas. Con todo, de lo anterior no se desprende que ahora un supuesto "sistema internacional" tenga finalmente el control completo de la dinámica de 
industrialización local, ya que el efecto paradójico del sistema tecnológico-institucional internacional que está cobrando vida en la actualidad es hacer que la movilización local "endógena" de recursos y capacidad para la industrialización, sea aún más importante como una condición para el éxito en el nuevo ámbito internacionalizado. Consecuentemente, el nuevo sistema "posfordista" requiere, más que nunca, que no hagamos a un lado las lecciones del análisis "postimperialista" expuesto en la sección precedente. Trataré ahora de dar más consistencia a estos argumentos.

El modelo de producción fordista fue instalado definitivamente en las economías capitalistas en los años de entreguerra ${ }^{\mathfrak{G}}$ y tuvo su mayor auge durante un periodo de pleno fordismo correspondiente más o menos al prolongado desarrollo intempestivo de la posguerra, después de] cual, en los años setenta y, en especial, en los ochenta, entró en un periodo de crisis, restructuración y reorganización espacial extensas que aún está lejos de haber terminado. El fordismo se basaba esencialmente en la producción en serie, aunque no debemos olvidar que, a todo lo largo del periodo fordista, siguieron prosperando otros tipos de actividad productiva (como la producción en lotes en pequeña escala y la industria artesanal especializada). En su aspecto clásico, el fordismo era apuntalado por grandes unidades de producción altamente capitalizadas consistentes ya sea en: a) procesos de flujo continuo, como en el caso de la producción de petroquímicos o de acero; ya en: b) procesos de línea de ensamble (y profundas divisiones técnicas de la mano de obra), como en los casos de las industrias automotriz, de aparatos domésticos o de maquinaria. La producción estaba orientada hacia una búsqueda insistente de economías de escala internas mediante la uniformación creciente de los productos, la introducción de procesos de producción rutinarios y el uso de equipo de capital de aplicación rígida. En consecuencia, tanto en el caso de la producción física por planta como en el de la productividad por trabajador, había una tendencia a aumentar firmemente con el tiempo. Las principales plantas de los sectores de punta fueron instaladas, y aún lo están, en el centro de redes de productores secundarios que proporcionaban los insumos y servicios físicos necesarios, basados a veces en procesos de mano de obra artesanal no fordistas. A medida que las actividades de producción selectas del sistema fordista alcanzaron la madurez tecnoló-

\footnotetext{
${ }^{6}$ Debo gran parte de lo expuesto en esta sección a Storper y Scott (1988a); además, deseo agradecer la intervención de Allen Scott en la formulación de algunos de los argumentos aducidos, si bien la responsabilidad por las conclusiones extraidas de ellos me corresponde únicamente a mí.
} 
gica, se tendió al establecimiento de plantas sucursales para llevar a cabo esas actividades y, después, a descentralizarlas en busca de mano de obra más barata, primero en las periferias nacionales y luego en las internacionales. Tal es el modelo que países como Brasil y México intentaron instalar dentro de sus fronteras.

Con todo, hacia finales de los años sesenta y principios de los setenta, la hegemonía de ese modelo de industrialización se vio amenazada. La saturación del mercado y el proceso de descentralización espacial total que surgió como respuesta a la militancia obrera estaban creando en el interior de los países del centro altos niveles de desempleo y descensos en la producción, mientras que, en el exterior, la competencia de Japón, y entre Estados Unidos y Europa Occidental, estaba reduciendo drásticamente los mercados nacionales de estas dos últimas regiones. El resultado neto fue que se intensificaron la restructuración y la racionalización in. dustriales en los países del centro, lo cual provocó más cierres de plantas, más descentralización y mayor desempleo. En ese contexto de estancamiento económico, productividad en descenso y competencia extranjera, las bases fiscales del estatismo benefactor keynesiano del hemisferio norte comenzaron a desmoronarse, como lo manifestó la prolongada crisis de estancamiento con inflación de los años setenta. Los choques petroleros'no hicieron más que sumarse a esas calamidades. La baja de las tasas de crecimiento del mundo desarrollado se combinó con las limitaciones internas de ese modelo de desarrollo en el Tercer Mundo (descritas en la primera parte de este trabajo] para producir una espectacular crisis de estancamiento con inflación en gran parte de América Latina y apareció el problema de la deuda externa como una de sus principales manifestaciones. Hacia finales de los años setenta, el modelo de industrialización fordista y los convenios macroeconómicos y políticos con él relacionados entraron en desorden permanente, tanto en el hemisferio norte como en el sur.

En estos últimos años del decenio de los ochenta, las condiciones de la competencia capitalista son muy diferentes de las existentes hace dos decenios. Las tasas de penetración cruzada en el mercado internacional son consistentemente más altas, en especial entre los países avanzados, y aun cuando la proporción de los mercados del mundo desarrollado representada por los bienes manufacturados provenientes del Tercer Mundo no ha aumentado de manera apreciable durante este periodo, las cantidades absolutas comprendidas - y con ellas su importancia relativa para los países productores del Tercer Mundo- han aumentado considerablemente. En resumen, la disputa y las posibilidades de disputa por los mercados respecto a una amplia gama de bienes 
y servicios son mucho mayores que lo que fueron en el apogeo de la producción en serie. Esta situación de disputa por el mercado ha renovado la clásica competencia de precios capitalista y estimulado mayores y más rápidas diferenciaciones y sustituciones de productos.

En contraste directo con los altos niveles de estabilidad del mercado, con la uniformación de los productos y con la rutina de los procesos, características todas de la producción en serie fordista, el nuevo régimen tecnológico-institucional de producción que se está adueñando progresivamente de la posición central en las economías capitalistas se basa en la "flexibilidad". Cuando hablamos de sistemas de producción flexibles, nos referimos a formas de producción caracterizadas por una capacidad muy desarrollada para cambiar fácilmente de un proceso, o configuración de producto, a otro (flexibilidad dinámica), y para ajustar rápidamente las cantidades de producción al alza o a la baja en el corto plazo sin efectos demasiado nocivos sobre los niveles de eficacia (flexibilidad estática). Estos dos tipos de flexibilidad se logran a través de una variedad de aspectos entrecruzados del sistema de producción. En una empresa, la flexibilidad puede lograrse mediante la utilización de equipo y maquinaria de uso general no específico (a menudo programablè) o mediante procesos de mano de obra artesanal. En el campo de las relaciones interempresas, el principal medio para lograr la flexibilidad es la fragmentación de la organización de los procesos de producción (desintegración horizontal y vertical) mediante la creación de una profunda división social de la mano de obra entre las empresas, lo cual facilita poder efectuar cambios rápidos en las combinaciones verticales y horizontales entre los productores de un sistema. En muchos aspectos institucionales diferentes, la subcontratación es la principal forma adoptada por esa división de la mano de obra, y es tal que el sistema de producción se convierte a menudo en una red de producción (Piore y Sabel, 1984). Por lo tanto, esas redes se encuentran integradas al proceso de producción, pero son externas a las empresas. Podemos referirnos a esas características como la "externalización" de la actividad de producción en el periodo del posfordismo y, asimismo, hacer notar que, como resultado de esas mismas características, las economías de escala externas del sistema de producción en su conjunto se ven intensificadas. Además, los mercados de mano de obra asociados a los sistemas de producción flexibles se caracterizan también por altas tasas de rotación y por la proliferación de trabajo de medio tiempo y temporal, así como doméstico.

Como consecuencia de las tendencias antes descritas, las uni- 
dades de producción individuales de los sistemas de producción flexibles son generalmente menos especializadas y de menor tamaño que las unidades de producción en serie. Desde el punto de vista tecnológico, esas unidades son capaces de lograr una gran flexibilidad de producción en sus propias esferas de operación y, al mismo tiempo, esa flexibilidad se ve multiplicada por los efectos del sistema sobre la división social de la mano de obra, lo cual, a su vez, permite la formación y reformación de combinaciones interdependientes de productores. Como resultado de lo anterior, la diferenciación de productos aumenta y los mercados se vuelven aun más competitivos.

En resumen, a lo largo y ancho de la amplia gama de industrias, la sobrevivencia en los mercados capitalistas de finales de los años ochenta depende de que se cumplan tres condićiones fundamentales e interrelacionadas: a) los altos niveles de interpenetración hacen indispensables la especialización y la diferenciación de productos - los bienes se vuelven menos uniformes y se orientan más hacia áreas comerciales más reducidas-; b) los altos niveles de competitividad hacen que la flexibilidad de producción sea necesaria como un medio para facilitar cambios entre productos diferentes; $c$ ) estas dos últimas condiciones hacen improbable que los productos especializados encuentren sus mercados exclusivamente dentro de las fronteras de un solo país, incluso el más grande y más rico, por lo que una tercera condición es que los productos sean dirigidos cada vez más tanto hacia los mercados nacionales como hacia los internacionales y, por ende, que también satisfagan cada vez más los patrones internacionales de precio, diseño y calidad.

Existen condiciones históricas específicas del Tercer Mundo que definen las posibilidades de éxito de sus estrategias de industrialización. Por una parte, los altos niveles de la deuda externa exigen que los países aumenten sus niveles de exportaciones para obtener créditos en divisas con los cuales pagar el servicio de la deuda y poder continuar la compra de bienes extranjeros, como los insumos industriales. Aun cuando los niveles de la deuda no son altos, las exportaciones son muy importantes, ya que permiten la expansión de las fuerzas productivas a una tasa más alta que la que proveería la sola expansión del mercado nacional (incluso suponiendo que el estado de las relaciones de clase fuese tal que permitiera el crecimiento del mercado nacional), siempre y cuando las exportaciones no dependan de mantener la mano de obra barata a tal punto que el mercado nacional se vea deprimido en una mayor medida que lo que puede compensar el volumen de las exportaciones. Por supuesto, la principal tarea de la política de 
industrialización en su conjunto reside en la coordinación de esas dos tasas: la expansión de las exportaciones y el crecimiento del mercado nacional provocado por los salarios. En las condiciones actuales del mercado, esa coordinación sólo puede lograrse a través de una hábil combinación de mejoramiento continuo de las relaciones precios-comportamiento de los productos de un país, es decir, a través del mejoramiento de la calidad, lo cual permite, a su vez, el aumento de los precios sin la reducción de los salarios internos, y estos dos últimos factores deben ser apoyados por aumentos regulares en el factor productividad. Obsérvese, también, que el cambiar continuamente las características precio-comportamiento de un producto tiene su análogo en el carácter tecnológico del proceso de producción: estar equipado para hacer cambios en los productos implica una capacidad para adaptar la tecnología del proceso a las condiciones locales, dándole la vuelta así, en cierta medida, al problema de importar tecnologías "inadecuadas". Eso es exactamente lo que hicieron los japoneses en los años cincuenta, cuando, a pesar de lo pequeño de su mercado nacional, de la escasez de capital y de unà situación relativamente rica en mano de obra, desarrollaron su ahora famoso sistema de producción Toyota "flexible": adaptaron el equipo estadunidense importado a las condiciones, más exigentes, del medio ambiente japonés (Cosumano, 1985).

Aunque específicas de los países del Tercer Mundo, todas esas condiciones constriñen las estrategias de industrialización de esos países de diversos modos coincidentes, en gran medida, con las condiciones que enfrentaron las economías avanzadas al adaptarse al nuevo régimen tecnologicoinstitucional de industrialización flexible: a) deben orientarse, en cierto grado, hacia la exportación; b) deben buscar la diferençiación y la especialización de productos (no sólo debido a los caprichos de los mercados mundiales y a sus cada vez más especializadas estructuras comerciales, sino también debido a las propias necesidades de las naciones del Tercer Mundo de hacer subir la curva precios-comportamiento), y c) en virtud de las dos últimas condiciones, la flexibilidad de los procesos de producción es indispensable en la actualidad.

El desarrollo posfordista: "endógeno", pero ¿orjentado hacia el exterior?

Las estrategias de desarrollo para la industrialización posfordista son, por necesidad, diferentes de las propuestas para implantar las formas de producción fordistas. La producción en serie fordis- 
ta se caracterizaba, sobre todo, por la posibilidad de separar las actividades de concepción -en el sentido del diseño de productos y procesos y de planeación de la producción-de la ejecución, esto es, la actividad real de tomar insumos y transformarlos en productos finales. Hacia finales de los años sesenta, esa separación se había extendido a tres aspectos de los sistemas de producción en serie. Primero: se dio una considerable desespecialización de los obreros de la producción, de modo que éstos se vieron reducidos a realizar tareas de repetición interminable con muy poca o ninguna relación con la toma de decisiones, situación que fue hecha posible por la división técnica de la mano de obra y su acompañante, el aspecto rutinario de las tareas. Aunque el obrero industrial recibió la denominación de "semiespecializado", en realidad grandes proporciones de los trabajadores de las fábricas fordistas poseían muy pocas habilidades especializadas, y en su mayoría podían ser capacitados en cuestión de horas o unos cuantos días cuando mucho. Segundo, muchas fases del sistema de producción estaban aisladas de las actividades de concepción porque la uniformación de productos y el aspecto rutinario de los procesos posibilitaron altos niveles de mecanización mediante la utilización de equipo de capital específico (esto es, especializado. y cuasi automático). Este llamado estado de "madurez tecnológica" significó que numerosas fases del sistema de producción fordista se redujeran a operaciones "llave en mano" (esto es, proyectos de fabricación completos) que podían duplicarse en cualquier lugar que contara con la infraestructura urbana necesaria y la de mano de obra suficiente. Hacia principios de los años setenta, la separación de las actividades de producción de las de concepción se había extendido, más allá de la cadena de insumos, a muchos componentes. Tercero, como correlato de estos últimos puntos, la separación espacial de la ejecución de las actividades de concepción se fue desarrollando cada vez mejor con la madurez del sistema. En las primeras décadas de posguerra, las plantas sucursales empezaron a ser trasladadas de las regiones de producción centrales de las naciones avanzadas a las regiones periféricas de las mismas, como el sur estadunidense, y más tarde, también fue posible el desplazamiento de grandes secciones de la cadena secundaria de insumos; por ejemplo, en los años en que Brasil ingresó en su época de auge industrial en los setenta, no era tecnológicamente difícil establecer secciones muy grandes del sistema de producción fordista lejos de los países desarrollados, donde se seguía realizando una gran parte de las actividades de concepción. En efecto, las políticas de industrialización basadas en la sustitución de importaciones eran coincidentes con la lógica de 
organización y emplazamiento de la producción en serie fordista a nivel mundial.

Consecuentemente, la producción flexible contemporánea se basa, sobre todo, en cuatro grupos principales de industrias: a) las de alta tecnología selecta; b) las de producción en lotes basada en la especialización (con una demanda intensiva de mano de obra, por lo general); c) las de servicios al productor y financieros, y d) las de productos de consumo duraderos restructurados y las de producción de bienes de capital pesados. La lógica de organización y emplazamiento de la producción en serie fordista no es funcional en ninguna de ellas. Obsérvese que los tres primeros de esos grupos, ninguno de los cuales fue especialmente importante durante el apogeo de la producción en serie fordista, representan en la actualidad proporciones de empleo y producción en crecimiento continuo y sostenido en todas las economías avanzadas y están ganando terreno en varias economías en desarrollo.

A continuación menciono algunos aspectos de la profunda separación entre las actividades de concepción y las de ejecución característica de la desintegración de la producción en serie en producción flexible:

Primero

La flexibilidad dinámica reside en la adecuada movilización y organización de las redes de productores. La división flexible de la mano de obra entre las diferentes empresas define las posibilidades para introducir las innovaciones en los productos y en los procesos; además, las cuestiones y problemas prácticos generados constantemente por la interacción entre los diferentes productores ponen en movimiento, también constantemente, procesos informales de innovación y de cambio técnicos. Tomemos un ejemplo del mundo desarrollado: en el distrito de Sassuolo, en el centro de Italia, muchas de las innovaciones introducidas en el equipo especializado para la fabricación de azulejos se han visto estimuladas por la interacción entre los productores de azulejos (con sus necesidades) y los productores de equipo (con sus aptitudes especializadas) (Russo, 1985). Como resultado de esa interacción entre las empresas, Sassuolo ha llegado a ser no sólo el principal productor de azulejos, sino también un importante exportador de equipo de producción de azulejos. En una escala más pequeña, lo mismo parece ser cierto en el caso de los productores de textiles y fabricantes de equipo de Blumenau y Joinville, en el estado de Santa Catarina, en Brasil, o en el de los zapateros de Novo Hamburgo, en Rio Grande do Sul. En el proceso de realización de sus negocios a tra- 
vés de redes de.producción flexibles, los empresarios aprenden acerca de las múltiples facetas de producción pertenecientes al complejo industrial. El resultado es la formación de una cultura comercial local en la que se socializan formas prácticas de conocimiento sobre los procesos de producción y los mercados y en la que se pulen los gustos y sensibilidades respecto a los materiales y los diseños de productos. En algunos casos, el hecho de que el Estado proporcione una infraestructura educativa que sirva al complejo local puede hacer que la socialización de conocimientos útiles vaya más lejos. Visto de 'esta manera, el sistema de producción ya no es una compañía, sino una organización socioeconómica más amplia que crea y define oportunidades empresariales.

\section{Segundo}

La naturaleza de la organización del sistema de producción flexible crea problemas potenciales de coordinación económica y social que sólo pueden ser resueltos endógenamente, es decir, en el lugar. Los sistemas de producción flexibles constituyen colectividades de productores independientes cuyas relaciones tradicionales pueden ser mediadas en parte por mecanismos de mercado y un comportamiento de toma de decisiones individual, esto es, la compra y venta de relaciones. No obstante, es muy raro que, por sí solos, los mercados resuelvan los problemas de flexibilidad que surgen, por una parte, de la interdependencia provocada por la división de la mano de obra entre las empresas y, por la otra, de la multiplicación de centros independientes de toma de decisiones que acompaña a dicha interdependencia. Por lo tanto, en los sistemas de producción flexibles exitosos pueden crearse mecanismos de coordinación formal - como contratos y alianzas estratégicas- o nuevos tipos de organizaciones especializadas en la coordinación de sistemas - como el zaibatsu japonés o el impannatori italiano-. En general, unos y otros se vinculan estrechamente con la base regional del sistema de producción y, en muchos casos, se ven apoyados por políticas públicas.

Otro camino para llegar a la coordinación de los sistemas de producción es el surgimiento de relaciones de confianza informales; a través de ellas, y con el tiempo, los productores y una comunidad industrial aprenden respecto al comportamiento, la idiosincracia y la capacidad de otros empresarios. Sin embargo, como cimiento de las relaciones económicas, la confianza es una "espada de dos filos": cuando, por una parte, constituye una forma estrictamente privada de coordinación transaccional, como en el caso de las mafias y las aristocracias, puede retardar el desarrollo económico, ya 
que aquéllas, por definición, restringen el ingreso de nuevos productores; cuando, por otra parte, la confianza es generalizada y pública, sirve para apoyar las transacciones y reducir los costos de los productores establecidos, mientras que, al mismo tiempo, permite que nuevos productores ingresen a la comunidad (Gambetta; 1988). Tanto los sistemas de confianza informales como las agencias de coordinación formales sirven para incrementar el flujo de información a través del sistema de producción y, así, alientan la dinámica de innovación a la que antes nos referimos. Lo que queremos hacer destacar es que la administración de las fuerzas de producción flexibles depende, en medida creciente, de la generalización de relaciones sociales adecuadas, en el plano del propio sistema de producción.

Es bien sabido que esos mecanismos y hábitos "externalizados" de coordinación a menudo no están presentes en las economías del Tercer Mundo. Hirschman (1984), por ejemplo, cita el problema de muchas compañías de aviación de los países en desarrollo: una vez en el aire (esto es, una vez que la maquinaria empieza a moverse), son tan modernas como cualquier otra compañía; pero, en tierra, la situación es con frecuencia muy diferente. Muchos de los teóricos del polo de desarrollo no lograron comprender que la sola introducción de tecnología moderna no provocaría automáticamente el establecimiento de tecnologías o de relaciones sociales comparables, hacia arriba o hacia abajo de la cadena de producción. Sin embargo, al menos bajo el fordismo y por espectaculares que sean las interrupciones ocasionales en el servicio y mantenimiento de esos sistemas tecnológicos, pudieron implantarse "islas" tecnológicas modernas en gran escala en países en desarrollo cuyas relaciones sociales serían incompatibles en otras circunstancias. Necesariamente, como consecuencia de la extendida "internalización" de funciones (dentro de la gran empresa) que no podían ser confiadas al medio ambiente transaccional exterior, la estructura industrial resultante es muy oligopólica. No obstante, esta forma de fracaso comercial en gran escala no parece ser compatible con una producción flexible exitosa.

\section{Tercero}

La especialización de la mano de obra requerida para llevar a cabo la producción flexible es diferente en muchos sentidos a la que interviene en la producción en serie (Storper y Scott, 1988b). Sin duda, ciertas fases de los sistemas de producción flexibles continúan dependiendo de una mano de obra extremadamente no especializada y altamente explotada (en particular a niveles infe- 
riores del sistema de subcontratación]. Sin embargo, también parece ser que la proporción entre mano de obra no especializada y mano de obra especializada es menor en el sistema de producción flexible típico que lo que era en el sistema de producción en serie fordista: existen proporciones más altas de empresarios, técnicos, diseñadores de productos, coordinadores de sistemas, etcétera; además, a través de la facilidad de distribución de la mano de obra en el taller, necesariamente se da una gran flexibilidad en la forma de grupos de trabajo y categorías de trabajo más amplias. Por lo general, la flexibilidad interna del trabajo exige más habilidades polivalentes que las que exigía el desempeño del trabajo en las condiciones fordistas, y gran parte de ese capital humano es específico de las compañías, ya que requiere una interacción flexible en la empresa en torno a una mezcla cambiante de tareas y proyectos de producción. Aunque estas características puedan referirse únicamente a una minoría de la fuerza de trabajo del sistema de producción flexible, se trata, no obstante, de una minoría lo suficientemente medible como para hacer ver la necesidad de prestar una atención mucho más cuidadosa a la creación y producción de especialidades de mano de obra de lo que fue el caso en la producción en serie fordista (como lo atestiguan la preocupación actual por los sistemas de educación en países como Estados Unidos y Francia). Consecuentemente, no es posible transferir así como así la producción flexible a las fuerzas de trabajo sin educación ni especialización del Tercer Mundo; exige el desarrollo de numerosas especialidades prácticas de producción.

La producción flexible impone nuevas exigencias (y, sin duda alguna, nuevas dificultades) a los lugares que desean industrializarse, ya que requiere la movilización y el apoyo endógenos de recursos (mano de obra, por ejemplo) y la alimentación de relaciones sociales complejas (confianza, capacidad empresarial, etcétera). Paradójicamente, parece ser que, para poder dominar el concepto esencial para la ejecución exitosa de la producción flexible y así dar servicio a los mercados mundiáles, en la actualidad es necesario movilizar recursos y especialidades locales en mayor medida que antes. Es evidente que, en el pasado, los japoneses comprendieron esta paradoja y que, ahora, Corea y Singapur la están tomando en serio, en el momento en que intentan impulsarse hacia las filas de las potencias industriales con efectividad reconocida en los mercados mundiales. Es obvio que esa forma dis industrialización no es barata o fácil de desarrollar; no existe manera de legislar para dar vida al sistema de producción flexible imponiendo simplemente barreras comerciales, como intentó hacerlo Brasil con respecto a la industria de las computadoras 
Cuando mucho, este último tipo de política sólo puede asegurar que los productos desarrollados en otra parte sean copiados y producidos para el mercado nacional (a precios mucho más altos, por lo general), mientras que no necesariamente lleva a la innovación dinámica de productos que podría permitir la exportación ni a la innovación de procesos que permitiría reducir los precios y expandir los mercados nacionales. La creación de especialidades, la movilización de recursos sociales y la identificación de áreas comerciales factibles y no ocupadas implican inversiones de tiempo y recursos sociales, en gran escala y a corto plazo, con altos riesgos de fracaso y largos periodos de madurez y amortización.

El posfordismo, la urbanización y el desarrollo regional en el Tercer Mundo

Como hemos observado, la característica de todas las industrias de producción flexible es que constituyen redes de productores especializados en las que las empresas pequeñas y medianas cuentan con una amplia representación. En esos sistemas, la tendencia es que las empresas establezcan relaciones comerciales muy activas. Ahora bien, la realización de muchas de las transacciones es potencialmente muy costosa, ya que las mismas implican tiempos de entrega precisos, la renegociación constante de insumos y productos y el desarrollo e intercambio de una información cambiante y peculiar a cada una de ellas. Por lo tanto, la tendencia entre las empresas de las industrias de producción flexible es concentrarse geográficamente para reducir los costos y dificultades de sus transacciones y perfeccionar su acceso al contexto informativo y cultural del sistema de producción. La reagrupación geográfica de la demanda y oferta de mano de obra alienta también la flexibilidad y el reajuste de la producción; lo cual, a su vez, facilita la rotación acelerada, reduce los costos de la búsqueda de mercados de mano de obra tanto para los empleadores como para los trabajadores, permite que el proceso de socialización y capacitación se desarrolle con mayor facilidad y, por ende, fomenta la adaptación de los trabajadores a las tareas. Consecuentemente, el giro hacia la flexibilidad en la producción se ha visto caracterizado por una firme reconcentración geográfica de la producción y por el resurgimiento del fenómeno del distrito industrial; de ma nera similar, la aparición de la producción flexible ha desmentido las predicciones (comunes en los años setenta) respecto a la muerte de los sistemas de producción aglutinados densamente en un 
solo lugar. ${ }^{7}$ En los países desarrollados, por ejemplo, vemos que se está dando una mayor reconcentración de actividades de negocios y de servicios comerciales en Nueva York, Londres, Tokio y otras "ciudades mundiales" junto con grupos dependientes en ciertas zonas de sus áreas suburbanas periféricas. En la actualidad, la revitalizada industria basada en la artesanía tiene su más alto desarrollo en los grupos especializados de la Tercera Italia, pero también podemos encontrarla en Francia, Dinamarca, Estados Unidos y Alemania. En lugares como el Silicon Valley y el Orange County, en California, en la Ciudad Científica, al sur de París, en Dallas-Fort Worth, Texas, y en el eje CambridgeReading-Bristol, en Inglaterra, existen nuevos distritos industriales de alta tecnología, y la producción flexible de bienes de consumo duraderos restructurados, que alcanza su máximo desarrollo en Japón, adopta formas como la del denso complejo de producción de Toyota City. En Brasil, en las pequeñas ciudades de Santa Catarina, como Blumenau, y a lo largo del Valle de Itajaí, así como en un gran número de ciudades de Rio Grande do Sul y en la región occidental del estado de São Paulo, han hecho su aparición zonas incipientes de producción flexible; además, Brasil está tratando de desarrollar un distrito industrial de alta tecnología en Campinas, ubicada a aproximadamente $100 \mathrm{~km}$ de la ciudad de São Paulo.

El desarrollo de la producción flexible representa, a la vez, un peligro y una oportunidad para las políticas regionales de las naciones en vías de industrialización. Por una parte, no existe un proceso de descentralización uniforme de la producción del mundo desarrollado hacia el mundo subdesarrollado en el que puedan confiar para su futuro los países en vías de industrialización; la reconcentración geográfica de la producción significa que la ubicación de los centros de crecimiento se encuentra cada vez más en el propio mundo desarrollado. Por otra parte, las políticas de industrialización exitosas destinadas a hacer frente a las realidades del nuevo sistema tecnológico-institucional ofrecen la oportunidad, en algunos casos, de romper con los patrones de extrema superioridad de las metrópolis: La característica común de los sectores de producción flexible la constituye su relativa independencia de los tipos particulares de economías de aglomeración que se encuentran disponibles en los viejos centros de la industria fordista, es decir, la vinculación con los complejos de producción

\footnotetext{
7 Por supuesto, los distritos a que nos referimos no son independientes, sino que dependen de una más amplia y expansiva división del trabajo regional, nacional e internacional.
} 
en serie y el acceso a su mano de obra especializada concomitante. A ello se debe que tantas de las industrias de producción flexible del mundo desarrollado hayan crecido en lugares muy alejados de las zonas industriales importantes tradicionales de esos países [el Sunbelt de Estados Unidos, el sureste de Inglaterra, el sur de Francia, etcétera). Y quizá es también la razón por la que los ejemplos incipientes de producción flexible de Brasil estén todos muy lejos de la órbita de São Paulo. La apertura de la "ventana de oportunidades de ubicación" por la aparición de nuevos sectores y nuevos métodos de producción ofrece la posibilidad de reducir la concentración espacial de la industria.

Con todo, que nosotros sepamos, ni siquiera los países desarrollados cuentan con una fórmula política exitosa para crear o sostener tales complejos de producción flexible. Sí existe una abundante literatura académica sobre temas tales como políticas de "tecnópolis", regiones "creativas" y áreas de "alta calidad de la vida", cuyo propósito es identificar las claves para reproducir los sistemas de producción flexible én nuevos lugares; pero una gran parte de esa literatura carece de un contenido analítico específico, ya que la mayoría de sus autores confía en descripciones ad hoc de las características de las regiones industriales en crecimiento. La tela de esas listas puede cortarse prácticamente a la medida de cualquier situación (Scott y Storper, 1987), y el peligro reside en que esas fórmulas políticas simplistas serán pasadas a los países del Tercer Mundo por analistas que tienen puntos de vista muy equivocados, incluso respecto a los países desarrollados.

En esta era de producción flexible, las políticas de industrialización regional podrían ser enfocadas con mayor efectividad, sin duda alguna, mediante la formulación de políticas destinadas a: a) como antes dijimos, fomentar el establecimiento de relaciones intrincadas entre las empresas; b] promover y apoyar la coordinación de esas mismas relaciones a través de métodos tanto formales como informales, incluyendo la identificación de mercados adecuados; c) impulsar la creación de mano: de obra especializada local y la operación del mercado de mano de obra local, y d) llevar a cabo procesos adecuados de regulación social local, incluido el desarrollo de la confianza en las comunidades (Storper y Scott, 1988). Difícilmente puede decirse que tales políticas equivalen a la palabrería de las tradicionales políticas de desarrollo regional o planeación urbana, ya que las que nosotros proponemos no pueden consistir simplemente en complejos incentivos financieros (como en el caso, por ejemplo, del SUDENE de Brasil) ni en reglamentos (como en la mayoría de las políticas respecto al uso de la tierra urbana). Con ninguno de estos enfoques se puede realizar 
el trabajo necesario para establecer la producción flexible como un sistema de relaciones y transacciones sociales a través de la acumulación constante del conocimiento local y del desarrollo de la práctica social local, así como de la inversión de capital.

\section{Conclusión}

Durante algún tiempo, los fracasos del fordismo en el Tercer Mundo fueron atribuidos a una especie de "lógica que todo lo abarca" del imperialismo que, como lo ilustró atinadamente Lipietz (1986), se convirtió en "la bestia del apocalipsis" para muchos estudiosos del desarrollo, el enemigo externo de uso múltiple a cuyos pies podían arrojarse los problemas del Tercer Mundo. Ese momento en la teoría fue desafortunado, ya que, en las propias sociedades en desarrollo, provocó que la atención se alejara de la intrincada dinámica de las clases y la política y, más especialmente, del fracaso que significó no haber logrado complementar la producción fordista con unas estructuras de ingreso y una dinámica del crecimiento compatibles con ese sistema de producción. A medida que el fordismo es remplazado por un nuevo conjunto de estructuras de competencia e'intercambio mundiales, cobran vida los nuevos sistemas tecnológico-institucionales de producción. El remplazo del sistema tecnológico-institucional del fordismo por un régimen de flexibilidad en la producción, que está surgiendo gradualmente, introduce un conjunto de realidades nuevas que moldean la urbanización y el desarrollo económico regional en los países desarrollados, al igual que en el Tercer Mundo, y exige una revaluación precisa de conceptos y supuestos preconcebidos. En este momento es necesario, en gran medida, enfocar los problemas del desarrollo de una manera que sea posfordista y postimperialista.

\section{Bibliografía}

Azzoni, C. R., 1986, Indústria e Reversao de Polarizaçāo no Brasil. São Paulo, Universidade de São Paulo, Instituto de Pesquisas Economicas, Ensaios Económicos, p. 58.

Becker, D., R. Sklar et al., eds., 1987, Post-imperialism: International Capitalism and Development in the Late Twentieth Century. Boulder, Co., Rienner.

Cano, W., 1981. Raízes de Concentração Industrial em São Paulo. São Paulo, Queiroz Eda., 2a. ed. 
Cardoso, F. H., 1977, "The Consumption of Dependency Theory in the United States", en Latin American Research Review, 12, pp. 7-24.

Corbridge, S., 1988, "Marxism and Development Studies: Beyond the Impasse". Siracusa, Nueva York, Syracuse University, Dept. of Geography, manuscrito.

Cosumano, M., 1985. The Japanese Automobile Industry. Cambridge, Harvard/Belknap East Asian Studies.

Evans, P., 1979, Dependent Development: the Alliance of Multinational State and Local Capital in Brazil. Princeton, N. J.

Gambetta, D., 1988, "Mafia: the Price of Distrust", en Gambetta, D., ed., Trust. Oxford, Basil Blackwell, pp. 158-175

Hirschman, A., 1984, ensayo, en Seer, Pioneers of Development.

Lewis, W. A., 1954, "Economic Development with Unlimited Supplies of Labor", en Manchester Bulletin of Social and Economic Studies, 22, pp. 139-191.

Lipietz, A., 1986, "New Tendencies in the International Division of Labor: Regimes of Accumulation and Modes of Social Regulation", en Scott, A. J., y M. Storper, eds., Production, Work, Territory: the Geographical Anatomy of Industrial Capitalism. Boston, Allen and Unwin, pp. 16-40.

Piore, M., y C. Sabel, 1984, The Second Industrial Divide. Nueva York, Basic Books.

Russo, M., 1985, "Technical Change and the Industrial District: the Role of Interfirm Relations in the Growth and Transformation of Ceramic Tile Production in Italy", en Research Policy, 14, 4, pp. 329-343.

Scott, A. J., y M. Storper, 1987, "High Technology Industpy and Regiona] Development: A Theoretical Critique and Reconstruction", en International Social Science Review, 112, pp. 215-232.

Storper, M., 1984, "Who Benefits from Industrial Decentralization? Social Power in the Labour Market, Income Distribution and Spatial Policy in Brazil", en Regional Studies, 18, 2, pp. 143-164.

y A. J. Scott, 1988a, "The Geographical Foundations and Social Regulation of Flexible Production Complexes", en Wolch, J., y $\mathrm{M}$. Dear, eds., Territory and Social Reproduction, Londres, Allen and Unwin.

y A. J. Scott, 1988b, "Work Organization and Local Labour Markets in an Era of Flexible Production", Ginebra, International Labour Office, World Employment Programme, ponencias sobre análisis del mercado de trabajo y planeación del empleo (en preparación).

y R. Walker, 1989, The Capitalist Imperative: Territory Technology and Industrial Growth, Oxford, Basic Blackwell (en preparación) 